\title{
Aleias do Parque Botânico do Ecomuseu Ilha Grande: um registro da história botânica da Universidade do Estado do Rio de Janeiro
}

\author{
Parque Botânico do Ecomuseu Ilha Grande Alleys: a record of the botanical \\ history of the University do Estado do Rio de Janeiro
} \begin{abstract}
Marcelo Dias Machado Vianna Filho ${ }^{5}$, Ricardo Carneiro da Cunha Reis ${ }^{6}$, Vivianne Ribeiro Valença ${ }^{7}$
\author{
2 Bióloga. Universidade Federal do Rio de Janeiro (UFRJ), Brasil. E-mail: carlaygm@gmail.com \\ 3 Bióloga. Universidade do Estado do Rio de Janeiro (UERJ), Brasil. E-mail: nattacha.moreira@gmail.com \\ 4 Biólogo. Universidade do Estado do Rio de Janeiro (UERJ), Brasil. E-mail: marcelofcastilhori@gmail.com \\ 5 Doutor. Universidade do Estado do Rio de Janeiro (UERJ), Brasil. E-mail: marceloviannafilho@gmail.com \\ 6 Biólogo. Universidade do Estado do Rio de Janeiro (UERJ), Brasil. E-mail: rccreis@gmail.com
}

1 Professora. Universidade do Estado do Rio de Janeiro (UERJ), Brasil. E-mail: catia.callado@gmail.com

7 Museóloga. Universidade do Estado do Rio de Janeiro (UERJ), Brasil. E-mail: vivianne.valenca@gmail.com
\end{abstract}

Cátia Henrique Callado ${ }^{1}$, Carla Y Gubáu Manão일 ${ }^{2}$ Nattacha dos Santos Moreira ${ }^{3}$, Marcelo Fraga Castilhori ${ }^{4}$,

Recebido em: 05/07/2019 | Aprovado em: 02/04/2020

DOI: $10.12957 /$ interag.2019.53542

\section{Resumo}

Este trabalho apresenta a estruturação das aleias do Parque Botânico do Ecomuseu Ilha Grande (PaB), localizado na Ilha Grande, Angra dos Reis, Rio de Janeiro, Brasil. A denominação das aleias ressalta a história de seis importantes pesquisadores da biodiversidade brasileira que tiveram suas trajetórias acadêmicas, seja quanto à formação, atuação e/ou constituição de acervo científico, relacionadas à Botânica da Universidade do Estado do Rio de Janeiro (UERJ): Dr. Jorge Pedro Pereira Carauta, Dra. Margarete Emmerich, Dra. Graziela Maciel Barroso, Dr. Luiz Emygdio de Mello Filho, Dr. Heinrich Maximilian Friedrich Hellmuth Sick e Sra. Margaret Ursula Mee. Assim, as aleias não apenas demarcam os jardins temáticos e conduzem os visitantes pelo $\mathrm{PaB}$, mas também apresentam o papel efetivo da UERJ na história botânica do Brasil.

\begin{abstract}
This study reports the structuring of the alleys of the Parque Botannico do Ecomuseu IIha Grande (PaB), located in I/ha Grande, Angra dos Reis, Rio de Janeiro State, Brazil. The name of the alleys highlights the history of six notorius researchers of Brazilian biodiversity who had their academic trajectories, regarding their professional qualification, professional performance and/or contribution for scientific collection, related to the Botany of the Universidade do Estado do Rio de Janeiro (UERJ): Dr. Jorge Pedro Pereira Carauta, Dr. Margarete Emmerich, Dr. Graziela Maciel Barroso, Dr. Luiz Emygdio de Mello Filho, Dr. Heinrich Maximilian Friedrich Hellmuth Sick and Mrs. Margaret Ursula Mee. Thus, the alleys not only delimit the thematic gardens and lead visitors through the $P a B$, but also highlight the effective role of UERJ in botanical history of Brazil.
\end{abstract}

Palavras-chave: Jardins Botânicos, Museus de Ciências, Instituto Penal Cândido Mendes.
Keywords: Botanical Gardens, Science Museums, Instituto Penal Cândido Mendes

Área temática: Cultura. Linha de extensão: Divulgação científica e tecnológica. Patrimônio cultural, histórico e natural. Turismo. 


\section{Introdução}

A Botânica ou Biologia Vegetal é o ramo da Ciência voltado ao estudo da vida vegetal em seus mais variados aspectos e manifestações. A Ciência Botânica se dedica a identificar, descrever, compreender e catalogar as plantas, bem como suas estruturas, propriedades, processos de vida, doenças, impactos e interações com o ambiente. O estudo da Botânica tem destaque no Brasil em várias instituições superiores, como na UERJ, que desde sua formação apresenta papel de destaque nessa área da Biologia.

A história botânica da Universidade do Estado do Rio de Janeiro (UERJ) tem início no extinto Curso de História Natural, da então Universidade do Estado da Guanabara (UEG), que congregava as cátedras: Biologia Geral, Botânica, Zoologia, Geologia e Paleontologia, Mineralogia e Petrografia1. Esse curso contribuiu para a formação do atual Curso de Ciências Biológicas do Instituto de Biologia Roberto Alcantara Gomes e formou os primeiros botânicos da UERJ. Atualmente, nessa Instituição, a Botânica é abordada nos campos da pesquisa, ensino e extensão, sendo ensinada em cursos de graduação em Ciências Biológicas, presencial e à distância, e em cursos de pós-graduação, acadêmicos e profissionais. Entre as Unidades Acadêmicas nesta área do conhecimento, destacam-se pelo registro, catalogação e divulgação de plantas: o Herbário da Universidade do Estado do Rio de Janeiro (HRJ), o Herbarium Bradeanum (HB), o Herbário da Faculdade de Formação de Professores (RFFP), o Horto Botânico e o Parque Botânico do Ecomuseu Ilha Grande. A Botânica também está presente no paisagismo e, consequentemente, na preocupação com a estética acolhedora do ambiente universitário, através das plantas selecionadas e cultivadas nos jardins dos campi da UERJ. O Campus Francisco Negrão de Lima configura um exemplo desses jardins, que conta com projetos do botânico, professor da UERJ, Dr. Luiz Emygdio de Mello Filho, e dos paisagistas, Roberto Burle Marx e Fernando Chacel.

Como forma de registrar uma parte da história botânica da UERJ, o Parque Botânico do Ecomuseu Ilha Grande atribuiu às suas aleias os nomes de seis professores-pesquisadores que contribuíram para aumentar o conhecimento da flora brasileira, apresentando suas relações com a UERJ e, por vezes, com o próprio território da Ilha Grande. 


\section{O Parque Botânico do Ecomuseu Ilha Grande}

A participação da UERJ na Ilha Grande inicia-se com a desativação e implosão do Instituto Penal Cândido Mendes em 1994, com o termo de cessão de uso, no qual a Universidade passou a ocupar áreas e benfeitorias remanescentes dessa instituição carcerária. ${ }^{2,3}$ Hoje, neste local, estão instalados o Centro de Estudos Ambientais e Desenvolvimento Sustentável (CEADS) e o Ecomuseu Ilha Grande (ECOMIG). Essa última unidade acadêmica tem o objetivo de desenvolver ações voltadas à preservação, investigação, valorização e divulgação do meio ambiente, da história, memória e do patrimônio local, a partir das relações socioculturais das comunidades da Ilha Grande. É composta por quatro núcleos: Museu do Cárcere, Museu do Meio ambiente, Centro Multimídia e o Parque Botânico. ${ }^{4}$ No processo de criação e concepção do Ecomuseu Ilha Grande, surgiu a proposta inicial do Parque das Ruínas, posteriormente, chamado de Parque Botânico. Em 2002, o projeto para a implantação do Parque Botânico foi desenvolvido e, desde então, vem sendo organizado sob a direção da primeira autora deste trabalho, vinculada ao Departamento de Biologia Vegetal do Instituto de Biologia da UERJ.

A relação da Botânica ou Biologia Vegetal com as coleções de plantas vivas brasileiras remonta ao século XIX, quando os professores de agricultura eram incumbidos da direção, cultura e contabilidade financeira dos jardins botânicos que começavam a ser criados no País. Como atividades desses jardins, realizava-se a classificação das plantas, a aclimatação de plantas exóticas e o melhoramento das plantas nativas. ${ }^{5}$ Atualmente, as coleções de plantas vivas, sejam elas arboretos, hortos, parques ou jardins botânicos, diferem das primeiras coleções estabelecidas, por apresentarem múltiplas funções. A abordagem científica e o processo sistemático de registro de plantas atuais permitem transmitir informações científicas e culturais aos visitantes, bem como promover a melhoria efetiva das ações de conservação da flora e da educação ambiental. ${ }^{6,7}$

O Parque Botânico do Ecomuseu Ilha Grande teve seu projeto paisagístico ${ }^{8}$ estruturado em 2015 e conta com uma coleção de plantas nativas cientificamente identificadas, organizadas e documentadas, com finalidade de divulgação, estudo e registro do patrimônio florístico da Ilha Grande, de forma acessível ao público e servindo tanto à conservação quanto à educação, cultura e lazer. ${ }^{8}$ Essa coleção de plantas nativas está instalada no espaço interno da antiga Colônia Agrícola do Distrito Federal, posteriormente denominada Instituto Penal Cândido Mendes, no Campus UERJ Ilha Grande, Vila Dois Rios, Ilha Grande, Angra dos Reis, Rio de Janeiro, Brasil, sob as coordenadas geográficas 23¹0'34”S e 4422'39”W (Fig. 1). A visitação ao Parque Botânico 
propõe uma reflexão sobre a importância das plantas para o homem em diferentes épocas e divulga a história de ocupação da Ilha Grande. Instaladas sobre as ruínas do antigo presídio, as aleias e jardins temáticos proporcionam a contemplação de exemplares da flora (Fig. 2), incentivando diferentes olhares sobre a relação homem e ambiente. Assim, o acervo vivo do Parque Botânico vem sendo organizado em um cenário atrativo, no qual se associam elementos botânicos, históricos, socioculturais e conservacionistas.

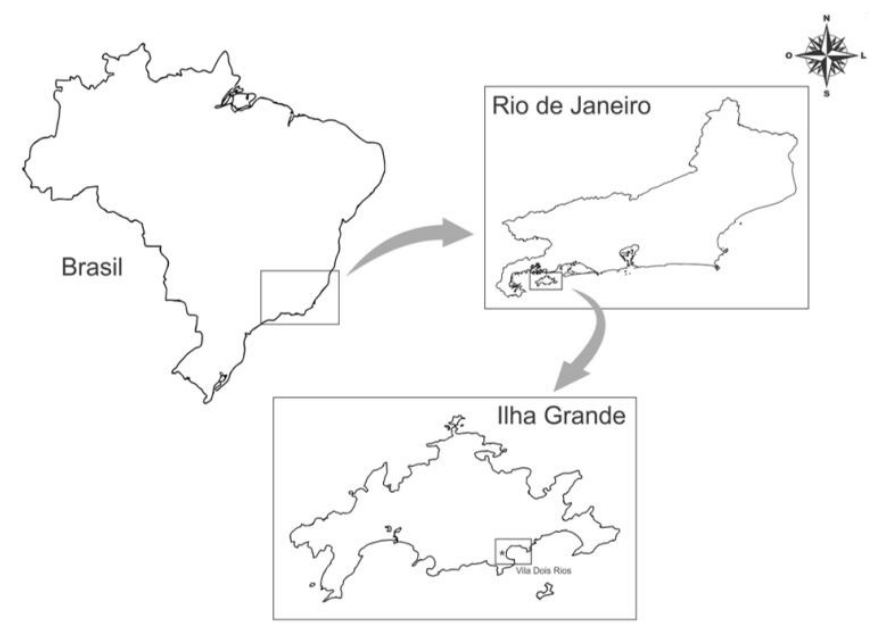

Figura 1
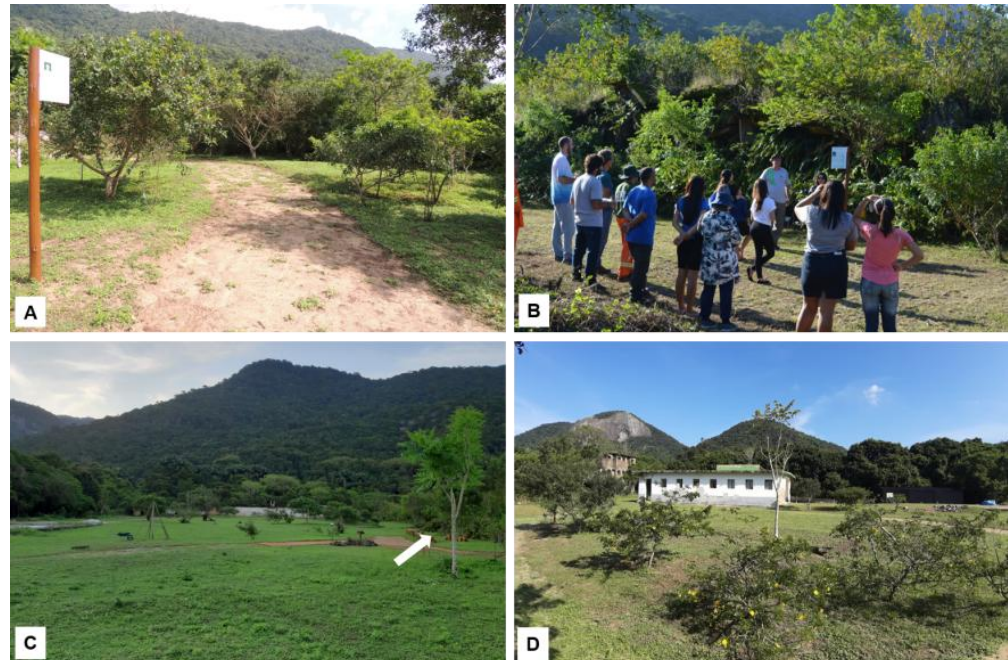

Figura 2 


\section{As Aleias do Parque Botânico do Ecomuseu Ilha Grande}

Uma aleia é uma alameda, caminho ou rua, ladeada por árvores, arbustos ou mesmo grades e estabelecida em jardins ou parques. No Parque Botânico, essas aleias determinam uma rede de caminhos (Fig. 3) e ao mesmo tempo resgatam e divulgam a história botânica relacionada à UERJ. Nesta etapa de trabalho, seis importantes pesquisadores tiveram seus nomes e parte de suas trajetórias acadêmicas apresentadas nas placas de identificação das aleias do Parque Botânico. A escolha dos homenageados considerou a importância desses pesquisadores para o avanço da Ciência Botânica e a relação dos mesmos com a UERJ, seja quanto à formação, atuação e/ou constituição de acervo, e, por vezes, com a própria Ilha Grande. Na abordagem adotada, o universo botânico tratado deixa de ser apenas o próprio ou particular da Ilha Grande para agregar e divulgar conhecimentos extra território.

A aleia de entrada é uma homenagem ao Dr. Jorge Pedro Pereira Carauta (19302013), que se graduou em História Natural pela Universidade do Estado da Guanabara (UEG), atual Universidade do Estado do Rio de Janeiro (UERJ) e tornou-se um renomado especialista no estudo das famílias Moraceae e Urticaceae. Além das grandes contribuições taxonômicas, era conhecido por ser um exímio escalador e pela grande capacidade de realizar expedições científicas em ambientes naturais, desenvolvendo uma maneira própria de ensinar botânica diretamente no campo. Trabalhou como analista de ecossistemas no extinto Herbário Alberto Castellanos do Instituto Estadual do Ambiente, além de ministrar aula no Curso de Pós-graduação em Ciências Biológicas (Botânica) do Museu Nacional da Universidade Federal do Rio de Janeiro. ${ }^{9}$ Entre outras espécies, nesta aleia é possível observar um exemplar de Ficus cyclophylla (Miq.) Miq. da família Moraceae, grupo taxonômico estudado pelo Dr. Carauta. A espécie é nativa na Ilha Grande, conhecida popularmente como gameleira-grande e integra a lista de espécies brasileiras vulneráveis à extinção. ${ }^{10}$ Seus indivíduos podem formar árvores que 
ultrapassam $30 \mathrm{~m}$ de altura, com flores na primavera e/ou verão, sempre após a queda das folhas, e os frutos são dispersos por animais, principalmente aves. ${ }^{11,12}$

A aleia Dra. Margarete Emmerich (1933-2015) é perpendicular à aleia Dr. Jorge Carauta e leva até a Casa de Produção de Mudas do Parque Botânico. A Dra. Margarete também se graduou em História Natural, pela UEG. Atuou no campo da taxonomia, como especialista na família Euphorbiaceae e, também, desenvolveu suas pesquisas no Museu Nacional da Universidade Federal do Rio de Janeiro. Foi uma das pioneiras no estudo da Etnobotânica brasileira, sendo um de seus principais focos de estudo a etnia Yawalapiti do Alto Xingu. ${ }^{13}$ Contribuiu para consolidação da Etnobotânica como Ciência que, por meio do registro histórico-científico, aproxima o conhecimento científico do saber tradicional e estimula soluções e estratégias para formulação de políticas voltadas à conservação ambiental e cultural.

A aleia Dra. Graziela Maciel Barroso (1912-2003) tem início na aleia Dra. Margarete Emmerich e percorre o Parque Botânico em toda a sua extensão. Conhecida como Primeira Dama da Botânica do Brasil, também se graduou em História Natural pela UEG. É reconhecida por sua excelência no estudo de grupos complexos da flora brasileira, como Asteraceae, Fabaceae e Myrtaceae. A Dra. Graziela Barroso também se destacou por intensa e contínua formação de recursos humanos, sendo a única brasileira a receber, nos Estados Unidos, a medalha Millenium Botany Award, entregue a botânicos dedicados à formação de pessoal. Desenvolveu suas pesquisas, principalmente, no Jardim Botânico do Rio de Janeiro, onde atuou até os 91 anos de idade. ${ }^{14}$

Partindo da aleia Dra. Graziela Maciel Barroso, a aleia Dr. Luiz Emygdio de Mello Filho (1913-2002) delimita um jardim de contorno circular que expõe plantas nativas da Ilha Grande com potencial ornamental. O Dr. Luiz Emygdio nasceu em Angra dos Reis e graduou-se em três diferentes áreas da Ciência: Medicina, Farmácia e História Natural. Tornou-se doutor pela UEG, onde foi professor de Botânica e o primeiro diretor do Instituto de Biologia. Foi também presidente da Fundação Brasileira para a Conservação da Natureza e diretor do Museu Nacional da Universidade Federal do Rio de Janeiro e da 
Fundação Parques e Jardins. Dedicou-se ao ensino da história da botânica e do paisagismo, em que ressaltava a necessidade da utilização de plantas nativas da flora brasileira como ornamentais. ${ }^{1,15}$

A aleia Dr. Hellmuth Sick (Heinrich Maximilian Friedrich Hellmuth Sick, 19101991) é paralela à aleia Dr. Jorge Pedro Pereira Carauta e localizada entre os escombros do antigo presídio e os jardins temáticos. Apresenta exemplares de Erythrina speciosa Andrews (Fabaceae), conhecida como mulungu-do-litoral, que foram plantados por visitantes do Parque Botânico. Este cientista foi um dos primeiros especialistas em aves tropicais e, por ser alemão, tornou-se preso político na Ilha Grande durante a 2a Guerra Mundial, quando fazia uma excursão científica pelo Brasil. No período de reclusão, estudou espécies de aves e de invertebrados, como cupins e formigas da Ilha Grande. Após sair da prisão, naturalizou-se brasileiro e trabalhou no Museu Nacional da Universidade Federal do Rio de Janeiro, destacando-se por descrever de maneira aprofundada as aves brasileiras. ${ }^{16}$ Embora o Dr. Hellmuth Sick não tenha sua formação acadêmica ligada à Botânica, esta relação foi estabelecida em suas expedições científicas, nas quais também se dedicava a observação e amostragem das plantas, contribuindo para o conhecimento de espécies novas para a Ciência. Sua relação com a Botânica da UERJ pode ser constatada no Herbarium Bradeanum, onde é possível encontrar os registros de algumas de suas coletas botânicas. Esta aleia representa o visitante, o que é externo à Ilha Grande, e relembra também o cárcere neste local.

Por fim, a aleia Margaret Mee (1909-1988) liga as aleias Dra. Margarete Emmerich e Dra. Graziela Maciel Barroso. Margaret Ursula Mee nasceu na Inglaterra e diplomou-se em pintura e design na cidade de Londres. Em 1952, mudou-se para o Brasil, lecionou na Escola Britânica de São Paulo e tornou-se ilustradora científica pelo Instituto de Botânica de São Paulo. Em 1964, iniciou efetivamente seus estudos, documentando e defendendo a flora e os ecossistemas brasileiros. A delicadeza de seus desenhos e pinturas, assim como a fidelidade à natureza, resultaram em um valioso registro das plantas nativas. Seu trabalho demonstra como Arte e Ciência podem se encontrar e se fundir 
harmoniosamente. Margaret Mee esteve sempre atenta em registrar todos os detalhes das plantas que retratava. ${ }^{17}$ É sob este aspecto que esta magnífica ilustradora se liga à Botânica da UERJ, onde é possivel encontrar, no acervo do Herbarium Bradeanum, o registro de seus ensaios e consultas ao botânico brasileiro Guido Frederico João Pabst sobre a autenticidade das formas e cores das plantas de suas aquarelas.

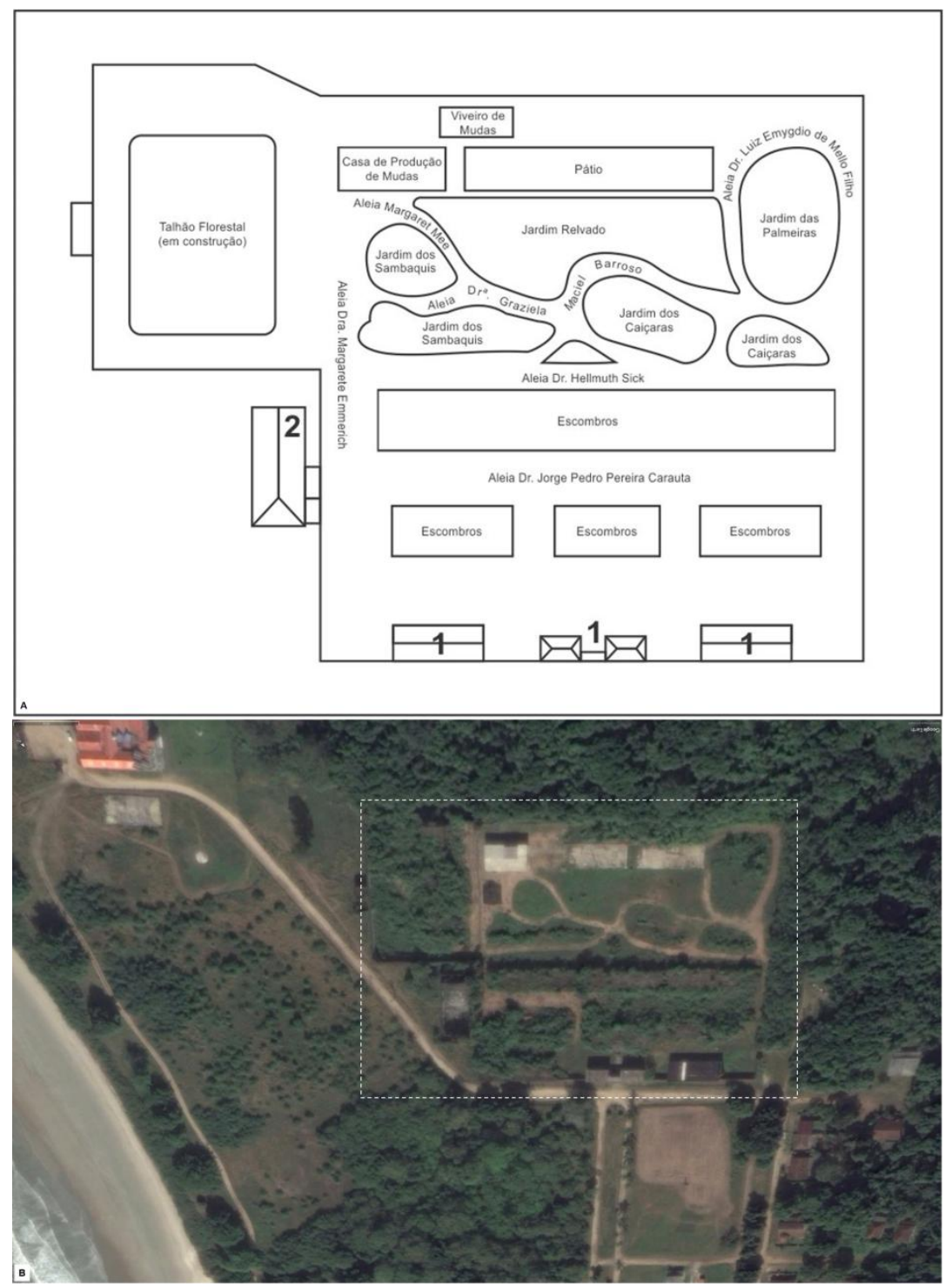

Figura 3 


\section{Conclusões}

O estabelecimento das aleias é uma necessidade de organização estrutural do Parque Botânico do Ecomuseu Ilha Grande. Todavia, a designação dessas aleias é mais um instrumento do $\mathrm{PaB}$ para a divulgação da botânica brasileira. Cabe ainda destacar, que com a evolução do acervo do $\mathrm{PaB}$, novas aleias podem ser formadas e devem seguir o mesmo critério de inclusão em relação à Ciência Botânica e a ligação com a Universidade do Estado do Rio de Janeiro.

\section{Agradecimentos}

Os autores agradecem ao ECOMIG, CEADS e Parque Estadual da Ilha Grande pelo apoio e infraestrutura disponibilizada para o desenvolvimento deste trabalho; ao CNPq pelo auxílio financeiro outorgado (Processo: 458122/2013-0); a Rodrigo Ashton pela confecção das figuras e aos Drs. Gelsom Rozentino e Ricardo Lima pela revisão dos textos das placas que nomeiam as aleias do Parque Botânico do Ecomuseu Ilha Grande.

\section{Referências}

1. Gomes, R.A. 1968. Apud: IBRAG. A Evolução Histórica do Instituto de Biologia. 2019. Disponível em <http://www.ibrag.uerj.br/index.php/o-ibrag/26-o-instituto/15a-evolucao-historica-do-instituto-de-biologia.html?tmpl=component\&print=1\&page=> . Acesso em 8 junho 2019.

2. Santiago, A.M.A.; Guimarães, C.; Nogueira, I.; Santos, M.S. \& Sant'anna, T. História da Ilha Grande e patrimônio cultural material e imaterial. In: M. Bastos \& C.H. Callado (Orgs.). O Ambiente da Ilha Grande. 1 ed. Rio de Janeiro: Universidade do Estado do Rio de Janeiro, 2009. p. 299-370.

3. Callado, C.H.; Bastos, M.; Manão, C.Y.G. \& Albarello, N. Ceads: um Centro de Pesquisa da Universidade do Estado do Rio de Janeiro e sua Contribuição para a Melhoria das Condições Humanas e Ambientais. Interagir, v. 26, p. 34-45, 2018. 
4. Lima, R.G.; Callado, C.H.; Faria, G.; Alevato, G.M.; Almeida, G.R.; Pereira, J.W.; Bastos, M.P.; Rosso, T.C.A. \& Castro, W.C. As especificidades dos ambientes insulares: meio ambiente e cultura - Estudo de caso do Ecomuseu Ilha Grande - UERJ. Interagir, v. 15, p. 11-18, 2010

5. Bediaga, B. O Jardim Botânico do Rio de Janeiro e as ciências agrárias. Ciência e Cultura, v. 62, p. 28-32, 2010.

6. Rede Brasileira de Jardins Botânicos. 2001. Normas internacionais de conservação para jardins botânicos. 1ª ed. Rio de Janeiro: Instituto de Pesquisas Jardim Botânico do Rio de Janeiro, 2001. $109 \mathrm{p}$.

7. Pereira, T.S. \& Costa, M.L.M.N. Os jardins botânicos brasileiros - desafios e potencialidades. Ciência \& Cultura, v. 62, p. 23-25, 2010.

8. Vianna-Filho, M.D.M.; Manão, C.Y.G.; Castilhori, M.F. \& Callado, C.H. O Parque Botânico do Ecomuseu Ilha Grande: planejamento e implantação In: Jardins históricos: intervenção e valorização do patrimônio paisagístico.1ed. Rio de Janeiro: Fundação Casa de Rui Barbosa, 2016, v.1, p. 333-346.

9. Cordeiro, S.Z.; Paiva, V.F \& Reis, K.C.G. Jorge Pedro Pereira Carauta. Universidade Federal do Estado do Rio de Janeiro. 2019. Disponível em http://www.unirio.br/ccbs/ibio/herbariohuni/patrono. Acesso em 13 junho 2019.

10. CNCFlora. Ficus cyclophylla in Lista Vermelha da flora brasileira versão 2012.2 Centro Nacional de Conservação da Flora. 2019. Disponível em <http://cncflora.jbrj.gov.br/portal/pt-br/profile/Ficus cyclophylla>. Acesso em 8 junho 2019.

11. Carauta, J.P.P. Ficus (Moraceae) no Brasil: Conservação e Taxonomia. Albertoa, v. 2, p. 1-365, 1989.

12. Pereira, R.A.S. Interações Antagonísticas de Figueiras e Psitacídeos. Natureza OnLine, v. 4, p. 25-29, 2006.

13. Boscolo, O.H.; Senna-Valle, L. \& Rocha, J.A. Contribuição de Margarete Emmerich para a botânica brasileira. Ethnoscientia, v. 3, p. 1-2, 2018. 
14. Morim, M.P. \& Peixoto, A.L. Graziela Maciel Barroso: uma trajetória na ciência botânica do século XX. Sociedade Botânica do Brasil. 2018. Disponível em <http://www.botanica.org.br/go_news.php?id=62> Acesso em 23 setembro 2018.

15. Museu Nacional. 100 Anos de Luiz Emygdio de Mello Filho. 2013. Disponível em <http://www.museunacional.ufrj.br/siteluiz/index.htm> Acesso em 23 setembro 2018 .

16. Gonzaga, L.P. In memorian: Helmut Sick. Ararajuba, v. 2, p. 107-115, 1991.

17. Silva, L.M. Margaret Mee e a Ilustração Científica. 2019. Disponível em $<$ http://www2.eca.usp.br/cms/index.php?option=com_content\&view=article\& $\mathrm{id}=66$ : margaret-mee-e-a-ilustracao-cientifica\&catid=14:folios\&Itemid=10> Acesso em 01 julho 2019. 